Tropical Journal of Pharmaceutical Research January 2016; 15 (1): 39-45

ISSN: $1596-5996$ (print); 1596-9827 (electronic)

(C) Pharmacotherapy Group, Faculty of Pharmacy, University of Benin, Benin City, 300001 Nigeria.

All rights reserved.

Available online at http://www.tjpr.org

Original Research Article

http://dx.doi.org/10.4314/tjpr.v15i1.6

\title{
Luteoloside Inhibits Proliferation of Human Chronic Myeloid Leukemia K562 Cells by Inducing G2/M Phase Cell Cycle Arrest and Apoptosis
}

\author{
Jun-Li Shao*, Hai-Rong Liang and Juan-Xiu Dai \\ Dongguan Key Laboratory of Environmental Medicine, School of Public Health, Guangdong Medical University, Dongguan, \\ Guangdong 523808, China
}

*For correspondence: Email: sjl@gdmc.edu.cn; Tel: 0086-759-22896573

Received: 1 October 2015

Revised accepted: 23 December 2015

\begin{abstract}
Purpose: To investigate the effects of luteoloside on the proliferation of human chronic myeloid leukemia K562 cells and whether luteoloside induces cell cycle arrest and apoptosis in K562 cells.

Methods: Luteoloside's cytotoxicity was assessed using a cell counting kit. Cell cycle distribution was analysed by flow cytometry after propidium iodide (PI) staining. Cell apoptosis was assayed with apoptosis detection kit and Hoechst staining followed by observation under a fluorescence microscope. The expression of cell cycle- and apoptosis-related proteins was examined by Western blot analysis.

Results: Luteoloside inhibited the proliferation of K562 cells in a dose- and time- dependent manner $\left(I C_{50}=30.7 \mu \mathrm{M}\right)$ with less toxicity in a normal human cell line $\left(I C_{50}=91.8 \mu \mathrm{M}\right)$. Moreover, antiproliferative effect of luteoloside was accompanied with G2/M phase arrest $(p<0.05$ or $p<0.01)$ and apoptosis ( $p<0.01$ or $p<0.001$ ). Further studies revealed that the expression level of cyclinB1 was down-regulated by luteoloside treatment. Furthermore, luteoloside treatment also increased proapoptotic protein Bax expression and decreased anti-apoptotic protein Bcl-2 expression.

Conclusion: These results suggest that the inhibitory effect of luteoloside on K562 cell proliferation is associated with inducing G2/M phase arrest and apoptosis, and that luteoloside is worth further studying for anticancer potential.
\end{abstract}

Keywords: Luteoloside, Myeloid leukemia, Proliferation, Cell cycle arrest, Apoptosis, Anticancer

Tropical Journal of Pharmaceutical Research is indexed by Science Citation Index (SciSearch), Scopus, International Pharmaceutical Abstract, Chemical Abstracts, Embase, Index Copernicus, EBSCO, African Index Medicus, JournalSeek, Journal Citation Reports/Science Edition, Directory of Open Access Journals (DOAJ), African Journal Online, Bioline International, Open-J-Gate and Pharmacy Abstracts

\section{INTRODUCTION}

Chronic myeloid leukemia (CML) is a malignant hematological disease characterized by the deregulated growth of myeloid leukemia cells in the bone marrow and their accumulation in the blood. The incidence of CML is 1 - 2 cases per 100,000 adults, and CML accounts for approximately $15 \%$ of newly diagnosed cases of leukemia in adults [1]. Furthermore, CML is still one of the most difficult malignant hematological diseases to treat. The current commonly used chemotherapies are often accompanied with various side-effects, such as anemia, hemorrhage, infection, fever, enlargement of the liver, spleen and lymph nodes, and ostealgia [2]. Therefore, it is required to find new anticancer agents with increased efficacy and decreased toxicity.

Plant-derived compounds have attracted considerable attention in cancer chemoprevention and treatment because of their high efficiency and safety [3]. Luteoloside, a 
natural flavone subclass derived from plants, has exhibited a series of biological activities, such as potential antibacterial and antifungal [4], free radical scavenging [5], anti-oxidative capabilities $[6,7]$, protection effects against doxorubicininduced cardiotoxicity [8], and anticarcinogenic potential against colon carcinogenesis [5] and hepatocellular carcinoma $[9,10]$, etc. However, the effects of luteoloside on blood malignancy have not been reported.

Here we carried out a study on the cytotoxicity of luteoloside against human CML K562 cells. K562 cells were derived from CML patient carrying a BCR-ABL mutation. The mutation gives poor response to treatment by deregulated apoptosis, proliferation and differentiation [11]. Since an important mechanism for anticancer agents is to trigger apoptosis [12] and cell cycle arrest [13] in cancer cells while disturbing their proliferation, we evaluated the effects of luteoloside on cell cycle and apoptosis together with cell growth of K562 cells. To further explain the underlying mechanism of luteoloside effects, the expression of cell cycle- and apoptosis-related regulatory proteins were assessed in K562 cells treated with luteoloside.

\section{EXPERIMENTAL}

\section{Cell culture, antibodies and chemicals}

Leukemia cell $\mathrm{K} 562$ and human normal cell HUVEC12 were cultured in RPMI-1640 and DMEM media respectively. All of them were cultured in the media supplemented with $10 \%$ fetal bovine serum at $37{ }^{\circ} \mathrm{C}$ in a humidified $5 \%$ $\mathrm{CO}_{2}$ atmosphere. Antibodies against cyclinB1, $\mathrm{Bax}$ and $\mathrm{Bcl}-2$ were purchased from Cell Signaling (Boston, USA). NAPDH antibody and horseradish peroxidase-conjugated secondary antibody were purchased from Beyotime Biotechnology (Beijing, China). Cell Counting Kit8 (CCK-8) and annexinV-FITC apoptosis detection kit were purchased from DoJindo (Kumamoto, Japan).

RNase A, propidium iodide (PI), dimethyl sulfoxide (DMSO) and Hoechst 33342 were purchased from Sigma-Aldrich (St. Louis, USA). RIPA lysis buffer and BCA protein assay kit were purchased from CWBIO Biotechnology (Beijing, China). Luteoloside was purchased from Shanghai Winherb Medical Technology Co., Ltd. (Shanghai, China). Luteoloside was dissolved in DMSO at 25, 50, 100 and $200 \mathrm{mM}$ as stock solutions and stored at $-20{ }^{\circ} \mathrm{C}$, and diluted 1000 times with media as work solution when used.

\section{Cell growth inhibition assay}

HUVEC12 cells were seeded into 96 well microtiter plate $\left(3 \times 10^{3}\right.$ cells per well $)$, and allowed to attach for $16 \mathrm{~h}$. Then media were exchanged with fresh media containing luteoloside at the indicated concentrations. K562 cells were seeded into 96 well microtiter plate ( 3 $\times 10^{3}$ cells per well) together with various concentrations of luteoloside. Cells treated with $0.1 \%$ DMSO were used as controls. After treatment for 48 and $72 \mathrm{~h}$, the cytotoxicities of luteoloside against K562 and HUVEC12 cells were assessed using CCK-8 assay. Briefly, CCK8 reagent $(10 \mu \mathrm{L})$ was added to each well and the cells were incubated for $2 \mathrm{~h}$ at $37{ }^{\circ} \mathrm{C}$. Absorbance was then analysed on a microplate reader (BioTek Synergy2) at $450 \mathrm{~nm}$. All experiments were done in triplicate.

\section{Cell cycle analysis}

K562 cells were treated with luteoloside for $48 \mathrm{~h}$ at concentrations of 50 and $200 \mu \mathrm{M}$ or for 24 and $48 \mathrm{~h}$ at $50 \mu \mathrm{M}$. At the end of the treatment, the cells were collected by centrifugation $(1000 \mathrm{rpm}$, $5 \mathrm{~min}$ ), washed twice with ice-cold PBS, and fixed in $75 \%$ ethanol at $-20{ }^{\circ} \mathrm{C}$ overnight. Then, cells were washed twice with ice-cold PBS containing $0.2 \%$ TritonX-100, and stained with DNA-staining solution $(50 \mu \mathrm{g} / \mathrm{mL} \mathrm{PI}$ and 100 $\mu \mathrm{g} / \mathrm{mL}$ RNase A in PBS) in the dark for $30 \mathrm{~min}$ at room temperature. Finally, cells were analysed by a flow cytometer (Becton Dickinson, CA, USA). All experiments were in duplicate and repeated three times.

\section{Detection of apoptosis}

K562 cells were treated with luteoloside at different concentrations ( 50 and $200 \mu \mathrm{M}$ ) for 48 or $72 \mathrm{~h}$. Then, apoptosis was assayed with annexinV-FITC apoptosis detection kit following the manufacturer's instructions. Briefly, cells were harvested by centrifugation, washed twice in PBS and re-suspended in binding buffer. AnnexinV-FITC and PI were then added to the cell suspension. After incubation for $15 \mathrm{~min}$ in the dark at room temperature, cells were diluted with binding buffer again and the ratio of apoptotic cells was measured within $1 \mathrm{~h}$ with a flow cytometer. All experiments were measured in duplicate and repeated three times.

Hoechst 33342 staining was performed to detect the alterations of nuclear morphology of K562 cells after luteoloside treatment. Hoechst 33342 was added to the treated cells at a final concentration of $10 \mu \mathrm{g} / \mathrm{mL}$, and the cells were incubated for another $10 \mathrm{~min}$ at $37{ }^{\circ} \mathrm{C}$. The 
stained cells were then observed to examine the degree of nucleus condensation under a fluorescence microscope equipped with standard excitation filters (Nikon, Japan).

\section{Western blot analysis}

Cells treated with luteoloside at the indicated concentrations and for different incubation time were harvested, washed twice with ice-cold PBS and lysed in ice-cold RIPA buffer for $10 \mathrm{~min}$ on ice. The lysates were centrifuged at $12,000 \mathrm{rpm}$ for $10 \mathrm{~min}$ at $4{ }^{\circ} \mathrm{C}$, and supernatants were collected. Protein concentrations were determined with BCA protein assay kit. Equal amounts of protein sample $(30 \mu \mathrm{g})$ were fractionated on SDS-PAGE, and electrotransferred onto PVDF membranes (Millipore). After blocking with $5 \%(\mathrm{w} / \mathrm{v})$ non-fat dry milk in PBS for $1 \mathrm{~h}$ at room temperature, the membranes were incubated overnight at $4{ }^{\circ} \mathrm{C}$ with the specific primary antibodies with a 1:1000 dilution. The membranes were washed and then further incubated with horseradish peroxidaseconjugated secondary antibody (1:1000) for $2 \mathrm{~h}$ at room temperature. After the membranes were washed again, the protein bands were visualized using a chemiluminescence imaging method (Pierce ECL Western Blot Substrate). All blots were repeated three times.

\section{Statistical analysis}

Data are presented as mean \pm standard deviation (SD). Significant differences between treated and untreated (control) cells were identified by Student's $t$ test. $P<0.05$ was considered statistically significant. $\mathrm{IC}_{50}$ (half maximal inhibitory concentration) values were calculated by regression analysis.

\section{RESULTS}

\section{Luteoloside inhibited the proliferation of K562 cells}

Anti-tumor drugs are often screened according to their cytotoxic effects on cell proliferation or viability. Therefore, we first investigated the effects of luteoloside on the proliferation of K562 cells exposed to luteoloside at various concentrations and for different time interval. As shown in Fig 1, luteoloside significantly inhibited the proliferation of $\mathrm{K} 562$ cells in a concentrationand time-dependent manner. When K562 cells were treated for $72 \mathrm{~h}$, the $\mathrm{IC}_{50}$ value was $30.7 \mu \mathrm{M}$. Moreover, there were smaller cytotoxic effects of luteoloside on the normal cell line HUVEC12 at the tested concentrations, with an $\mathrm{IC}_{50}$ value of $91.8 \mu \mathrm{M}$ for $72 \mathrm{~h}$ treatment.

\section{Luteoloside induced cell cycle arrest at G2/M phase}

To investigate the mechanism behind the inhibition of cell growth by luteoloside, cell cycle analysis was conducted by flow cytometry. As shown in Fig. 2A, the percentage of G2/M phase increased as the concentration of luteoloside increased in the $48 \mathrm{~h}$ treatment, which was 28.0 $(p<0.05)$ and $35.8(p<0.01)$ at 50 and $200 \mu \mathrm{M}$, respectively, while that of the untreated cells was only 20.4 .

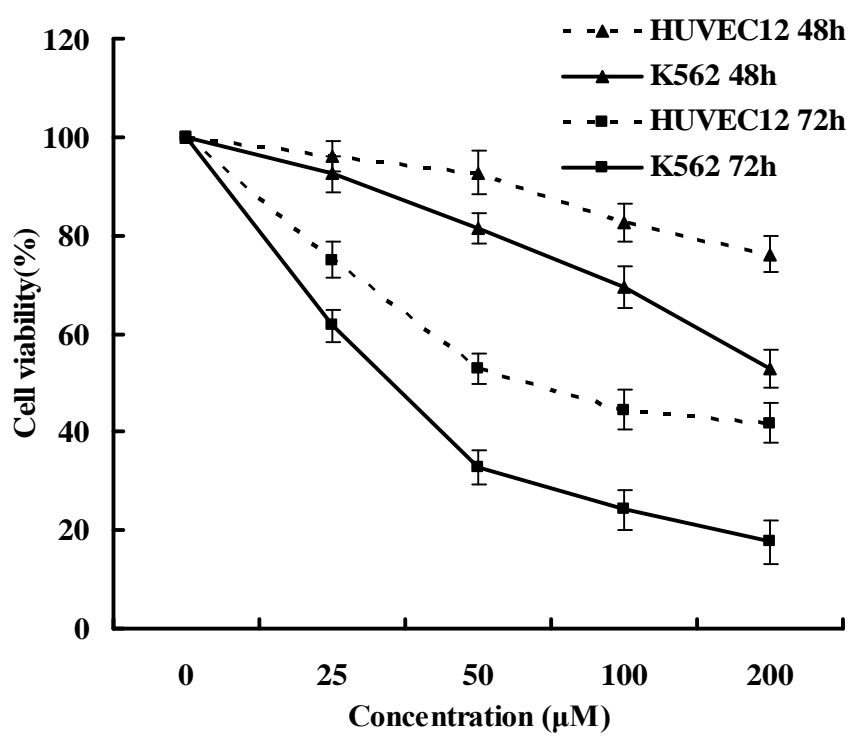

Figure 1: Effects of luteoloside on cell proliferation. Cells were treated with luteoloside at 25, 50, 100 and $200 \mu \mathrm{M}$ for 48 and $72 \mathrm{~h}$. Cell viability was determined using CCK-8 assay. Data are the mean \pm SD of three independent experiments 
Fig. 2B shows that the percentage of $G 2 / M$ phase increased with prolonged incubation time at $50 \mu \mathrm{M}$, which was $23.9(p<0.05)$ and $29.4(p$ $<0.01$ ) for 24 and $48 \mathrm{~h}$ treatment respectively, while that of the untreated cells was only 20.5 . These results demonstrate that luteoloside induces G2/M phase cell cycle arrest in K562 cells in a concentration- and time-dependent manner.

\section{Luteoloside-induced apoptosis}

Using flow cytometry after annexinV-FITC and PI staining, we evaluated whether luteoloside induced apoptosis in K562 cells. As it can be observed (Fig. 3A), luteoloside increased the number of total apoptotic cells 2.2-fold at $50 \mu \mathrm{M}$ and 3.4-fold at $200 \mu \mathrm{M}$ for $48 \mathrm{~h}$ treatment and 3.1 -fold at $50 \mu \mathrm{M}$ and 6.0 -fold at $200 \mu \mathrm{M}$ for $72 \mathrm{~h}$ treatment. Therefore, apoptotic effects of luteoloside on K562 cells were in a dose- and time-dependent manner. Since cell apoptosis is accompanied with alterations of nuclear morphology, Hoechst 33342 staining was performed to detect alterations of nuclear morphology of K562 cells treated with luteoloside. As shown in Fig 3B, the nuclei of the control cells were uniformly stained, while the nuclei of the treated cells showed the typical characteristics of apoptosis, including condensed chromatin and punctate apoptotic bodies.

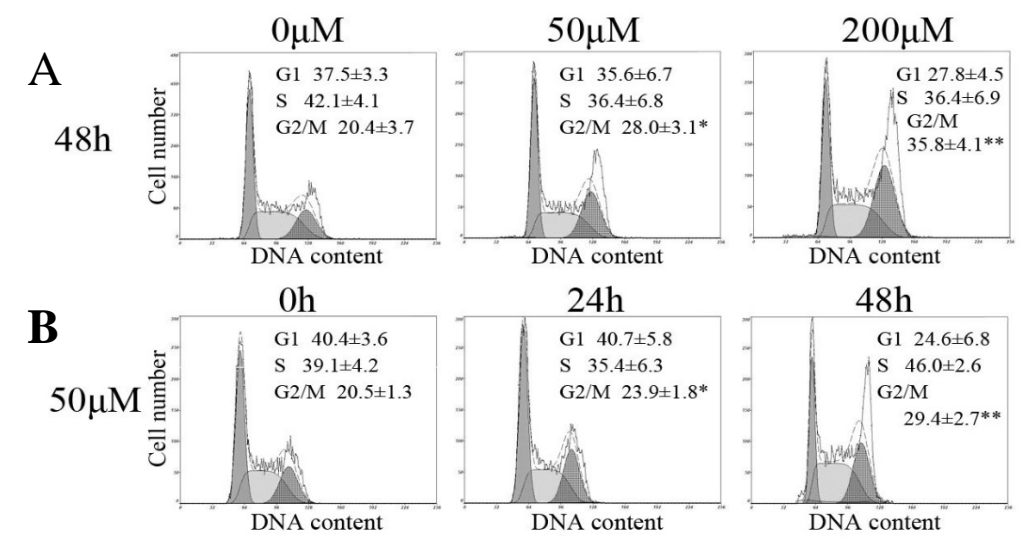

Figure 2: Effects of luteoloside on cell cycle distribution of K562 cells. (A) K562 cells were treated with 50 and $200 \mu \mathrm{M}$ luteoloside for $48 \mathrm{~h}$. (B) K562 cells were treated with $50 \mu \mathrm{M}$ luteoloside for 24 and $48 \mathrm{~h}$. Treated cells were harvested and fixed in $75 \%$ ethanol. Then, the cells were stained with $\mathrm{PI}$ and analysed using a flow cytometer. A representative profile is shown for each treatment. The data are the mean \pm SD of three independent experiments. ${ }^{*} p<0.05$ and ${ }^{* *} p<0.01$ indicate statistically significant differences versus control group

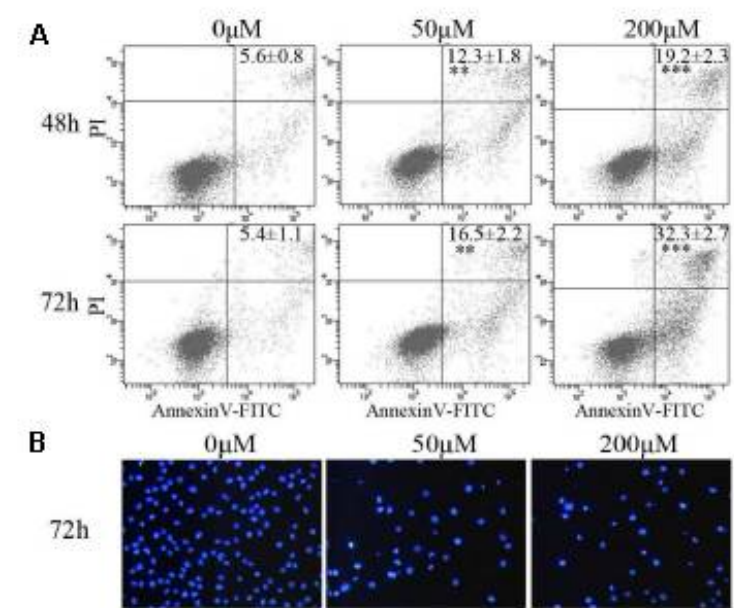

Figure 3: Effects of luteoloside on apoptosis of K562 cells. (A) K562 cells were treated with 50 and $200 \mu \mathrm{M}$ luteoloside and harvested at 48 and $72 \mathrm{~h}$. The cells were then analysed by flow cytometry after annexinV-FITC and PI staining. The data are the percentages of early and late apoptosis/necrosis cells (upper plus lower right quadrants), expressed as the mean $\pm S D$ of three independent experiments. Significant differences between treated cells and control cells are indicated as ${ }^{* *} p<0.01$ and ${ }^{* * *} p<0.001$. (B) The cells treated with luteoloside were stained with Hoechst 33342 and observed under a fluorescence microscope. Representative results from three independent experiments are shown 
Luteoloside decreased cyclinB1 and Bcl-2 expression and increased Bax expression

Expression of the intracellular proteins related to G2/M phase arrest and apoptosis, such as cyclinB1, Bax and Bcl-2, was investigated to understand the mechanisms by which luteoloside inhibited K562 cells. As shown in Fig 4, the expression of cyclinB1 decreased in response to luteoloside treatment. The expression of the proapoptotic protein Bax was upregulated markedly, while the expression of the anti-apoptotic protein Bcl-2 was suppressed by luteoloside at the indicated concentrations and for the incubation time.

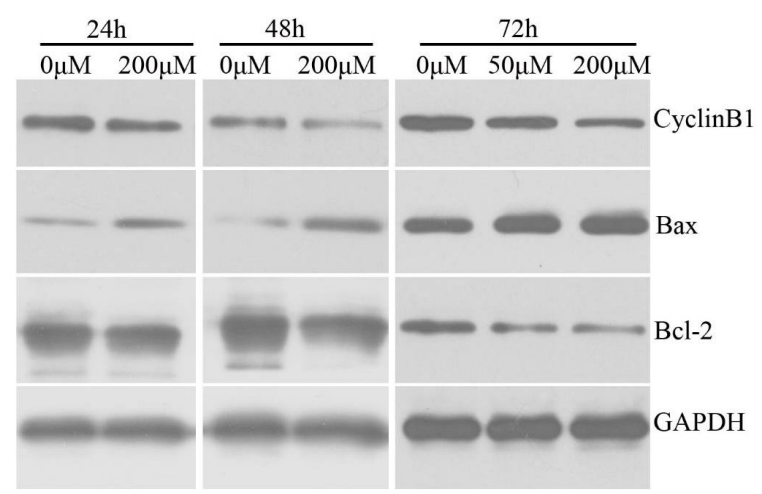

Figure 4: Effects of luteoloside on cell cycle- and apoptosis-related protein expression in K562 cells. Cells were treated with luteoloside at the indicated concentrations for 24, 48 and $72 \mathrm{~h}$. Protein samples of the treated cells were subjected to Western blot analysis with anti-cyclinB1, Bax and Bcl-2 monoclonal antibodies. The levels of GAPDH served as internal control. Representative results from three independent experiments are shown

\section{DISCUSSION}

Since rapid growth due to deregulated cell cycle and reduced apoptosis is the important property of cancer cells, inhibiting proliferation by arresting cell cycle progression and promoting apoptosis has been an important strategy for anticancer drugs and chemo-preventive agents $[12,15-17]$. In this study, it is revealed that luteoloside can induce cell cycle arrest and apoptosis concomitantly with growth suppression of K562 cells. Thus, luteoloside has exhibited its anti-cancer effects not only on solid tumors $[5,9,10]$ but also on blood malignancy.

Considering the side-effects of the current chemotherapy drugs, it is required to conduct research on agents with high efficiency and low toxicity. In this study, luteoloside showed high cytotoxicity on K562 cells in a dose- and time- dependent manner with an $\mathrm{IC}_{50}$ of value of 30.7 $\mu \mathrm{M}$ for $72 \mathrm{~h}$ treatment. In addition, toxicity evaluation on the normal cell HUVEC12 revealed only minor decrease in cell viability at the tested concentrations with an $\mathrm{IC}_{50}$ of $91.8 \mu \mathrm{M}$ for $72 \mathrm{~h}$ treatment. In contrast, luteoloside suppressed proliferation of several hepatocellular carcinoma cells [10], colon carcinoma cell COLO 320 DM and normal cell VERO [5] also in a dose- and time-dependent manner with $\mathrm{IC}_{50}$ of about 75 , 112.4 and $724 \mu \mathrm{M}$, respectively, for $72 \mathrm{~h}$ incubation. Therefore, the cytotoxic effects of luteoloside on K562 are in high efficiency and specific to some extent.

Reproducing healthy cells is strictly regulated due to rigorously and accurately controlled cell cycle. Control of the cell cycle is driven by the cyclin-dependent kinases (CDKs). Cells cannot enter $S$ phase or $M$ phase without CDK activation. In order to become catalytically active, CDKs need to bind to a cyclin subunit that acts as an activator [15]. CyclinB1 is one of main cyclins involved in the regulation of the transition from $G 2$ to $M$ phase during the cell cycle. Modification subsequent to CDK1 binding to cyclinB1 causes activation of the CDK1/cyclinB1 complex and drives the cell into mitosis [18]. CylclinB1 is synthesized initially in late G1 phase and its content reaches a maximum in G2 phase. It was reported that some natural or chemical compounds that cause $\mathrm{G} 2 / \mathrm{M}$ phase arrest involved the down-regulation of cyclinB1 $[19,20]$. In the present study, luteoloside treatment increased the cell number at G2/M phase, as confirmed by flow cytometry. Western blot analysis further revealed the decreased expression of cyclinB1. These results indicate that down regulation of cyclinB1 may contribute to luteoloside-induced G2/M arrest in K562 cells.

Apoptosis, also known as programmed cell death, plays an important role in the regulation of development and homeostasis [21,22]. Cells undergoing apoptosis always show a series of characteristics including cell shrinkage, membrane blebbing, chromatin condensation, DNA fragmentation and, eventually, cellular breakdown into apoptotic bodies [23].

Apoptosis occurs through two main pathways: the extrinsic pathway, known as death receptor pathway, and intrinsic pathway, known as mitochondria pathway [24]. Mitochondria play a central role as the key control point in the mitochondria pathway. Bcl-2 family proteins, outer mitochondrial membrane proteins, have been well known as crucial regulators in the mitochondria pathway. Based on function, they are divided into two subclasses, pro-apoptotic 
Bcl-2 family proteins (Bax, Bak and Bad, etc.) and anti-apoptotic Bcl-2 family proteins (Bcl-2, $\mathrm{Bcl}-\mathrm{xL}$ and $\mathrm{Mcl}-1$, etc.). Whether mitochondria pathway is activated depends on the balance between pro- and anti-apoptotic Bcl-2 family proteins. High expression of pro-apoptotic Bcl-2 family proteins can enhance the permeability of outer mitochondrial membrane and the consequent translocation of cytochrome $\mathrm{c}$ from the mitochondria to the cytoplasm, which provokes the downstream apoptotic events, while the anti-apoptotic Bcl-2 family proteins oppose the pro-apoptotic Bcl-2 family proteins [25-27]. Increasing $\mathrm{Bax} / \mathrm{Bcl}-2$ ratio often accompanies phytochemical-induced apoptosis through mitochondrial pathway in cancer cells [28-30].

In this study, luteoloside treatment triggered cell death of K562 cells by inducing apoptosis, which was confirmed by an increase in the percentage of total apoptotic cell population and the condensation or fragmentation of chromatin/ nuclei after luteoloside treatment. Further study displayed that the pro-apoptotic protein $\mathrm{Bax}$ increased and the anti-apoptotic protein Bcl-2 decreased after luteoloside treatment. These results indicate that luteoloside-induced apoptosis is mediated by Bax and Bcl-2 through the mitochondrial pathway. However, it cannot be excluded that the extrinsic apoptotic pathway is involved in the luteoloside-induced apoptosis based on the present results.

It should be noted that future studies are required to investigate more molecular events responsible for luteoloside-induced cell cycle arrest and apoptosis.

\section{CONCLUSION}

Despite its preliminary characteristics, this study indicates that luteoloside has antitumor potentials due to its growth inhibitory effect, G2/M phase arrest and inducement of apoptosis. Furthermore, decreased cyclinB1 expression may contribute to G2/M phase cell cycle arrest. Besides, up-regulation of pro-apoptotic molecule Bax and down-regulation of anti-apoptotic molecule Bcl-2, demonstrate that intrinsic apoptotic pathway is one of the pathways through which luteoloside-induced apoptosis occurs.

\section{ACKNOWLEDGEMENT}

This work was supported by grants from the education department of Guangdong province (no. 2012LYM_0067), the Dongguan key Laboratory of Environmental Medicine, the
Science and Technology Department of Zhanjiang City (no. 2014B01148) and Guangdong Medical University (nos. M2014006, 1057112025).

\section{REFERENCES}

1. Jabbour E, Kantarjian H. Chronic myeloid leukemia: 2014 update on diagnosis, monitoring, and management. Am J Hematol 2014; 89(5):547-556.

2. Zhang $N$, Wang $D$, Zhu $Y$, Wang J, Lin $H$. Inhibition effects of lamellarin D on human leukemia K562 cell proliferation and underlying mechanisms. Asian Pac J Cancer Prev 2014; 15(22):9915-9919.

3. Cragg GM, Newman DJ. Plants as a source of anticancer agents. J Ethnopharmacol 2005; 100(1-2):72-79.

4. Chiruvella KK, Mohammed A, Dampuri G, Ghanta RG, Raghavan SC. Phytochemical and Antimicrobial Studies of Methyl Angolensate and Luteolin-7-O-glucoside Isolated from Callus Cultures of Soymida febrifuga. Int $J$ Biomed Sci 2007; 3(4):269-278.

5. Baskar AA, Ignacimuthu S, Michael GP, Al Numair KS. Cancer chemopreventive potential of luteolin-7-Oglucoside isolated from Ophiorrhiza mungos Linn. Nutr Cancer 2011; 63(1):130-138.

6. Sun $X$, Sun $G B$, Wang $M$, Xiao J, Sun XB. Protective effects of cynaroside against $\mathrm{H}(2) \mathrm{O}(2)$-induced apoptosis in H9c2 cardiomyoblasts. J Cell Biochem 2011; 112(8):2019-2029.

7. Song YS, Park CM. Luteolin and luteolin-7-O-glucoside strengthen antioxidative potential through the modulation of Nrf2/MAPK mediated HO-1 signaling cascade in RAW 264.7 cells. Food Chem Toxicol 2014; 65:70-75

8. Yao $H$, Shang Z, Wang $P$, Li S, Zhang $Q$, Tian $H$, Ren D, Han X. Protection of Luteolin-7-O-Glucoside Against Doxorubicin-Induced Injury Through PTEN/Akt and ERK Pathway in H9c2 Cells. Cardiovasc Toxicol 2015.

9. Hwang YJ, Lee EJ, Kim HR, Hwang KA. Molecular mechanisms of luteolin-7-O-glucoside-induced growth inhibition on human liver cancer cells: G2/M cell cycle arrest and caspase-independent apoptotic signaling pathways. BMB Rep 2013; 46(12):611-616.

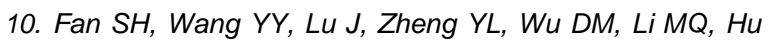
$B$, Zhang ZF, Cheng $W$, Shan $Q$. Luteoloside suppresses proliferation and metastasis of hepatocellular carcinoma cells by inhibition of NLRP3 inflammasome. PLoS One 2014; 9(2):e89961.

11. Skorski T. Genomic instability: The cause and effect of $B C R / A B L$ tyrosine kinase. Curr Hematol Malig Rep 2007; 2(2):69-74.

12. Fulda $S$. Targeting apoptosis for anticancer therapy. Semin Cancer Biol 2015; 31:84-88.

13. Shcherba $M$, Liang $Y$, Fernandes $D$, Perez-Soler $R$, Cheng $H$. Cell cycle inhibitors for the treatment of NSCLC. Expert Opin Pharmacother 2014; 15(7):9911004. 
14. Chen $C$, Wang JS, Qin D, Yang $Y, Y u$ YY, Fang Q. [The effect of retrovirus-mediated $\mathrm{HO}-1$ gene on chronic myeloid leukemia resistance cell K562/A02 apoptosis induced by nilotinib]. Zhonghua Xue Ye Xue Za Zhi 2012; 33(5):383-387.

15. Dibb M, Ang YS. Targeting the cell cycle in esophageal adenocarcinoma: an adjunct to anticancer treatment. World J Gastroenterol 2011; 17(16):2063-2069.

16. Skladanowski A, Bozko P, Sabisz M. DNA structure and integrity checkpoints during the cell cycle and their role in drug targeting and sensitivity of tumor cells to anticancer treatment. Chem Rev 2009; 109(7):29512973.

17. Evan GI, Vousden KH. Proliferation, cell cycle and apoptosis in cancer. Nature 2001; 411(6835):342-348.

18. Dai QS, Liu W, Wang XB, Lu N, Gong DD, Kong LY, Guo QL. NCPMF-60 induces G2/M cell cycle arrest and apoptosis in human hepatocellular carcinoma HepG2 cells. Anticancer Drugs 2011; 22(1):46-57.

19. Chelsky ZL, Yue P, Kondratyuk TP, Paladino D, Pezzuto JM, Cushman M, Turkson J. A Resveratrol Analogue Promotes ERKMAPK-Dependent Stat3 Serine and Tyrosine Phosphorylation Alterations and Antitumor Effects In Vitro against Human Tumor Cells. Mol Pharmacol 2015; 88(3):524-533.

20. Lohberger B, Kretschmer N, Bernhart E, Rinner B, Stuendl N, Kaltenegger $H$, Kahl S, Bauer R, Leithner A. 25-O-acetyl-23,24-dihydro-cucurbitacin $F$ induces cell cycle G2/M arrest and apoptosis in human soft tissue sarcoma cells. J Ethnopharmacol 2015; 164:265-272.

21. Curtin JF, Cotter TG. Live and let die: regulatory mechanisms in Fas-mediated apoptosis. Cell Signal 2003; 15(11):983-992.
22. Hengartner MO. The biochemistry of apoptosis. Nature 2000; 407(6805):770-776.

23. Denault JB, Boatright K. Apoptosis in Biochemistry and Structural Biology. 3-8 February 2004, Keystone, CO, USA. IDrugs 2004; 7(4):315-317.

24. Fulda S, Debatin KM. Extrinsic versus intrinsic apoptosis pathways in anticancer chemotherapy. Oncogene 2006; 25(34):4798-4811.

25. Kelly PN, Strasser A. The role of Bcl-2 and its prosurvival relatives in tumourigenesis and cancer therapy. Cell Death Differ 2011; 18(9):1414-1424.

26. Chipuk JE, Moldoveanu T, Llambi F, Parsons MJ, Green DR. The BCL-2 family reunion. Mol Cell 2010; 37(3):299-310.

27. Martinou JC, Youle RJ. Mitochondria in apoptosis: BCl-2 family members and mitochondrial dynamics. Dev Cell 2011; 21(1):92-101.

28. Kim CD, Cha JD, Li S, Cha IH. The mechanism of acacetin-induced apoptosis on oral squamous cell carcinoma. Arch Oral Biol 2015; 60(9):1283-1298.

29. Li PP, He W, Yuan PF, Song SS, Lu JT, Wei W. Celastrol induces mitochondria-mediated apoptosis in hepatocellular carcinoma Bel-7402 cells. Am J Chin Med 2015; 43(1):137-148.

30. Lin CC, Lee MH, Lin JH, Lin ML, Chueh FS, Yu CC, Lin JP, Chou YC, Hsu SC, Chung JG. Crude extract of Rheum palmatum $L$. Induces cell cycle arrest $S$ phase and apoptosis through mitochondrial-dependent pathways in U-2 OS human osteosarcoma cells. Environ Toxicol 2015; doi: 10.1002/tox.22105. 